\title{
DESENVOLVIMENTO DE NANO E MICROPARTÍCULAS DE ACETATO DE CELULOSE PARA SISTEMAS DE LIBERAÇÃO CONTROLADA DE ANTI-INFLAMATÓRIOS NÃO ESTERÓIDES
}

\section{DEVELOPMENT OF NANO AND MICROPARTICLES OF CELLULOSE ACETATE FOR CONTROLLED RELEASE SYSTEMS OF NONSTEROIDAL ANTI-INFLAMMATORY}

\author{
Mirele Costa Neves ${ }^{1}$, Thelma S. P. Cellet ${ }^{2}$, Adriano Lopes Romero ${ }^{1}$, \\ Rafaelle Bonzanini Romero ${ }^{1}$. \\ ${ }^{1}$ Universidade Tecnológica Federal do Paraná - UTFPR, Departamento de Química, \\ Campo Mourão, PR. ²Universidade Estadual de Maringá - UEM, Departamento de \\ Química, PR \\ e-mail: rbromero@utfpr.edu.br \\ Agência de fomento: Fundação Araucária e UTFPR.
}

RESUMO - Sistemas de liberação controlada de fármacos têm sido desenvolvidos visando inúmeras aplicações terapêuticas, dentre elas a diminuição dos efeitos colaterais de certos fármacos, destacando-se os antiinflamatórios não-esteróides. Diante do exposto, o presente trabalho tem como objetivo obter e caracterizar morfologicamente micro e nanopartículas de acetato de celulose $(\mathrm{AC})$, bem como estudar a potencialidade destes sistemas para liberação de anti-inflamatórios não-esteróides. Micro e nanopartículas de AC foram produzidas empregando a técnica de miniemulsão/evaporação do solvente. A influência do tipo de surfactante e agitação foram investigadas. Micrografias de microscopia eletrônica de varredura mostraram que foram obtidas micro e nanoesferas de AC com tamanho médio de $1000 \mu \mathrm{m}$ para as microesferas e de $100 \mathrm{~nm}$ para as nanoesferas. Estudos in vitro foram realizados em fluídos gástricos e intestinal simulados para investigar a liberação de paracetamol. Os resultados demonstraram que a liberação de anti-inflamatórios não-esteróides podem ser obtidas utilizando as microesferas desenvolvidas.

Palavras-chave: nano e micropartículas; acetato de celulose; anti-inflamatórios não-esteróides; sistemas de liberação.

Recebido em: 16/08/2017 Revisado em: 12/09/2017 Aprovado em: 02/10/2017
ABSTRACT - Controlled drug release systems have been developed for a number of therapeutic applications, including the reduction of side effects of certain drugs, especially nonsteroidal anti-inflammatory drugs. In this context, the present work aims to obtain and morphologically characterize micro and nanoparticles of cellulose acetate (CA), as well as to study the potential of these systems for the release of nonsteroidal anti-inflammatory drugs. The micro and nanoparticles of CA were produced using the miniemulsion/solvent evaporation technique. The influence of the type of surfactant and agitation were investigated. Scanning electron microscopy micrographs showed that were obtained micro and nanospheres of CA with average size about $1000 \mu \mathrm{m}$ for the microspheres and $100 \mathrm{~nm}$ for the nanospheres. In vitro studies have been carried out to investigate paracetamol release from microspheres in simulated gastric and intestinal fluids. The results demonstrated that 
controlled drug release of nonsteroidal anti-Inflammatory can be obtained using the microspheres developed.

Keywords: nano and microparticles; cellulose acetate; nonsteroidal antiinflammatory; drug delivery. 


\section{INTRODUÇÃO}

O uso abusivo e inadequado de fármacos anti-inflamatórios não esteroidais (AINEs) tais como paracetamol, naproxeno, ibuprofeno e ácido acetilsalicílico podem causar efeitos indesejáveis no organismo do indivíduo, visto que estes são medicamentos comercializados em drogarias sem prescrição médica.

Esses medicamentos são utilizados como analgésico e antipiréticos para aliviar dores e febre. No entanto não há uma preocupação por parte dos usuários quanto aos seus efeitos toxicológicos, que muitas vezes passam despercebidos ou até sem cuidado específico. Em casos de superdosagem, pode levar a efeitos tóxicos potenciais ocasionando uma encefalopatia hepática, falência hepática, e até mesmo a morte do indivíduo (ALLEN; POPOVICH; HOWARD, 2013).

O paracetamol é usado principalmente como analgésico e antipirético, apresenta uma baixa atividade antiinflamatória, mas tem provado eficácia clínica no alívio temporário de dores leves a moderadas associadas a resfriado comum, dor de cabeça, dor de dente, dores musculares, dor nas costas, dores leves de artrite, cólicas menstruais e redução da febre, sendo eficaz em bebês, crianças e adultos (FLOWER; VANE, 1972). No entanto, - uso do paracetamol por período prolongado pode desencadear toxicidade gastrintestinal em determinados pacientes, resultando em danos à mucosa, irritação e até mesmo hemorragia, quando tratados por via oral.

Neste contexto, a tecnologia de liberação controlada de fármacos representa uma das áreas da ciência, a qual envolve diferentes aspectos multidisciplinares e podem contribuir muito para o avanço da mesma. Os sistemas de liberação controlada de drogas vem revolucionando a indústria farmacêutica (produção de medicamentos), como também a indústria química (derivados de petróleo, agroindústria, indústria civil na produção de tintas), principalmente. A terapêutica moderna está baseada em um "design" racional de liberação controlada e alta especificidade de compostos de drogas.

Ao contrário das formas convencionais, de liberação imediata, os produtos de liberação modificada permitem a liberação retardada ou prolongada do fármaco, sendo muitos deles comprimidos ou cápsulas com revestimento entérico responsável por proteger as substâncias da degradação no suco gástrico (estômago), sendo o fármaco liberado apenas no intestino.

Produtos de liberação prolongada são desenvolvidos para liberar a substância ativa de modo controlado, em velocidade, tempo e local predeterminados, para alcançar e 
manter os níveis sanguíneos terapêuticos ótimos. Poucos fármacos de liberação imediata possuem uma longa ação que exija a administração de apenas uma dose diária que mantenha os níveis plasmáticos adequado e efeito terapêutico necessário (ALLEN; POPOVICH; HOWARD, 2013).

Os novos métodos para a administração de drogas se baseiam no encapsulamento de fármacos em veículos de entrega com diferentes formas (design), como nanocápsulas, microcápsulas, microesfera poliméricas ou proteicas. Estas, por sua vez, liberam a droga, já no interior do organismo, em pequenas e controláveis doses diárias, de acordo com a forma que foi projetada, durante dias, semanas ou meses, passando por vários fatores (eliminação de primeira passagem, ligações a proteínas, ligações a outros tecidos, entre outros), os quais podem interferir na biodisponibilidade, chegando na circulação sistêmica inalterado, pronto para interagir com seus sítios de ação (PARVEEN et al., 2012; SCHAFFAZICK et al., 2003).

Os sistemas de liberação controlada têm sido desenvolvidos visando inúmeras aplicações terapêuticas, e é uma das áreas mais promissoras na utilização de nanopartículas para vetorização de fármacos anticancerígenos (YOO, 2000; BRASSEUR; BRAULT; COUVREUR, 1991; COUVREUR; DUBERNET; PUISIEUX, 1995) e de antibióticos
(PINTO-ALPHANDARY;

ANDREMONT;

COUVREUR, 2000; FRESTA, 1995), principalmente através de administração parenteral, almejando uma distribuição mais seletiva dos mesmos e, assim, um aumento do índice terapêutico (LEGRAND et al., 2007).

Com relação à administração oral de nanopartículas, as pesquisas têm sido direcionadas especialmente à: a) diminuição dos efeitos colaterais de certos fármacos, destacando-se os anti-inflamatórios nãoesteróides (GUTERRES, 1995) que frequentemente causam irritação à mucosa gastrintestinal e b) proteção de fármacos degradáveis no trato gastrintestinal, como peptídeos (JUNG, 2000), proteínas (VILLA et al., 2002) ou hormônios (HIILLERY; TOTH; FLORENCE, 1996), aumentando a biodisponibilidade dos mesmos. Outro grande interesse nas nanopartículas é sua administração oftalmológica (CALVO; VILAJATO; ALONSO, 1996; LOSA et al., 1993), visando o controle da liberação, o aumento da biodisponibilidade ocular e a diminuição dos efeitos colaterais devido à absorção sistêmica de certos fármacos.

Os sistemas de administração oral estão em constante estudo por ser a via de maior aceitação por pacientes e médicos. A utilização de materiais poliméricos para uso na área da saúde está em constante crescimento, pois o mesmo tem a habilidade de auxiliar no processo produtivo e modular 
a liberação de forma lenta e gradual (ALLEN; POPOVICH; HOWARD, 2013). Nesse sentido, o acetato de celulose, que é um dos derivados da celulose, trata-se de um material promissor na utilização sistemas de liberação controlada, tanto na forma de filmes como na forma de nanopartículas (RIBEIRO et al., 2012).

As micropartículas são mais frequentemente preparadas utilizando a técnica de emulsão. A emulsão é um sistema contendo dois líquidos imiscíveis onde um dos quais está disperso no outro na forma de pequenas gotículas. Têm-se dois tipos de sistema: a emulsão água em óleo, em que a água é a fase dispersa e o óleo é a fase contínua, ou a emulsão óleo em agua, em que o óleo é a fase dispersa e a água a fase contínua. Pode-se também ter emulsões múltiplas do tipo $\mathrm{A} / \mathrm{O} / \mathrm{A}$ ou $\mathrm{O} / \mathrm{A} / \mathrm{O}$, que geralmente são preparadas em duas etapas. Por exemplo, em uma emulsão $A / O / A, a$ etapa consiste na formação da emulsão primária, água em óleo, sendo sequentemente re-emulsificada em outra fase aquosa (PAL, 2011).

Desta forma, este trabalho teve como objetivo obter micro e/ou nanopartículas a base de acetato de celulose (AC) e estudar a potencialidade destes sistemas para liberação controlada de anti-inflamatórios não-esteróides. As micro e/ou nanopartículas de AC foram produzidas empregando a técnica de miniemulsão/evaporação do solvente. A influência do tipo de surfactante e de agitação (agitação magnética ou ultrassônica) foram investigadas.

\section{METODOLOGIA}

Experimental. Na preparação das micro e/ou nanopartículas utilizou-se como matriz polimérica o acetato de celulose $(A C)$ da marca Sigma-Aldrich (CA, $M_{n}=30.000 \mathrm{~g} \cdot \mathrm{mol}^{-}$ ${ }^{1}$, grau de substituição de 2,5 ).

Método 1. Para a obtenção das micropartículas preparou-se três soluções. A fase orgânica (FO) foi preparada, sob agitação magnética por 1 hora, utilizando 20 $\mathrm{mL}$ de diclorometano e $300 \mathrm{mg}$ de AC. A fase aquosa (FA), foi preparada pela dissolução de $1500 \mathrm{mg}$ dos surfactantes Tween 80 ou poli(álcool vinílico) (PVA) em $100 \mathrm{~mL}$ de água, sob agitação magnética. A fase contendo o fármaco (FF) foi preparada pela dissolução de $30 \mathrm{mg}$ de Paracetamol em $2 \mathrm{~mL}$ de diclorometano. Após 30 minutos nessas condições, a fase orgânica e a fase com o fármaco foram adicionadas à fase aquosa e a agitação foi mantida até a evaporação total do solvente, à temperatura de 40 oC. Posteriormente foi realizada a filtragem e secagem para caracterização das partículas. Também foram realizados experimentos sem a introdução do fármaco, como estudo preliminar de liberação controlada. 
Método 2. A FO foi preparada, sob agitação magnética por 1 hora, utilizando 15,0 mL de diclorometano e $300 \mathrm{mg}$ de AC. A FA foi preparada pela dissolução de diferentes porcentagens em massa do surfactante PVA em $100 \mathrm{~mL}$ de água destilada sob agitação magnética. Em seguida, transferiu-se a FO à $F A$, que foram levadas a um ultrassom de ponta (Cole-Parmer 500, modelo EW-0471140), utilizando uma frequência de $20 \mathrm{kHz}$ por $5 \mathrm{~min}$, com ciclos de 30 segundos. Após a sonicação, aqueceu-se a solução a $40^{\circ} \mathrm{C}$ até a evaporação total do solvente. O produto foi separado por centrifugação, lavado com água e seco. Foram realizados três experimentos variando a porcentagem em massa do surfactante: Exp.1: 1500 mg de PVA; Exp.2: $500 \mathrm{mg}$ de PVA e Exp.3: $150 \mathrm{mg}$ de PVA.

\section{Caracterização das Amostras}

Caracterização Morfológica. A morfologia das amostras foram analisada por Microscopia Eletrônica de Varredura (MEV) utilizando um microscópio da marca Shimadzu, modelo SS550 Superscan, operando com voltagem de aceleração de 15 $\mathrm{kV}$ e intensidade de corrente de $30 \mu \mathrm{A}$. A superfície das amostras foram previamente cobertas com uma fina camada de ouro. $O$ tamanho médio das amostras foi determinado pelo software Size Meter.
Liberação controlada. Após a obtenção das partículas carregadas com fármaco, $100 \mathrm{mg}$ do material foi introduzido dentro de uma membrana de diálise. Posteriormente esta membrana foi adicionada à $10 \mathrm{~mL}$ de uma solução soluções tampão de pH 1,2 (fluido de simulação gástrica) ou $\mathrm{pH} 7,4$ (fluido de simulação intestinal). Esta suspensão formada foi mantida em um banho com temperatura controlada de 37 으. Alíquotas de $3 \mathrm{~mL}$ foram recolhidas, em intervalos específicos, e em seguida, as leituras de absorção foram realizadas a $240 \mathrm{~nm}$, por meio de um espectrofotômetro UV-Vis (T70+, marca $P G$ Instruments). Posteriormente as alíquotas foram devolvidas para dentro do frasco contendo as soluções. As concentrações de paracetamol liberadas a partir das partículas foram determinadas a partir de uma curva analítica, correlacionando a absorção versus concentração do fármaco utilizado.

\section{RESULTADOS}

No método 1 as micropartículas de $A C$ foram obtidas variando o tipo de surfactante. Para as amostras obtidas utilizando o surfactante Tween 80 , as imagens de microscopia eletrônica de varredura, Figura 1, mostram a formação de poucas partículas, desformes, de caráter esférico, com diferentes distribuição de diâmetro, Figura 1(a). Já, as micropartículas de AC contendo o fármaco, verificou-se a formação de esferas 
mais homogêneas e compactas, Figura 1(b).

Para as micropartículas obtidas utilizando

PVA como surfactante, observou-se a sendo elas mais uniformes e com diâmetro menores (Figura 2) quando comparado as obtidas com Tween 80.

formação de várias placas e algumas esferas,

Figura 1 - Micrografias de MEV das amostras obtidas pelo Método 1 utilizando o surfactante Tween 80: (a) pura e (b) com paracetamol.
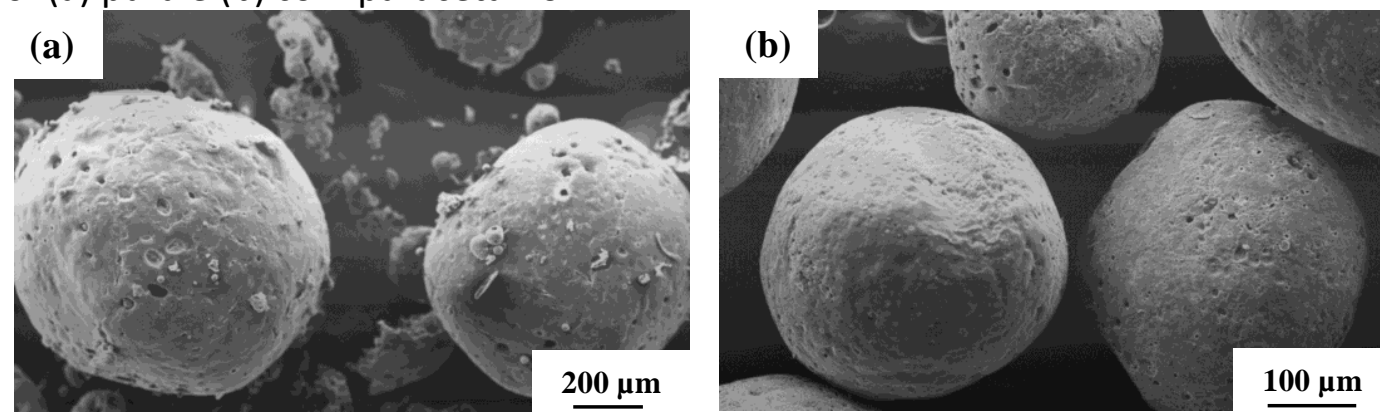

Figura 2 - Micrografias de MEV das amostras obtidas pelo Método 1 utilizando o surfactante PVA: (a) pura e (b) com paracetamol.
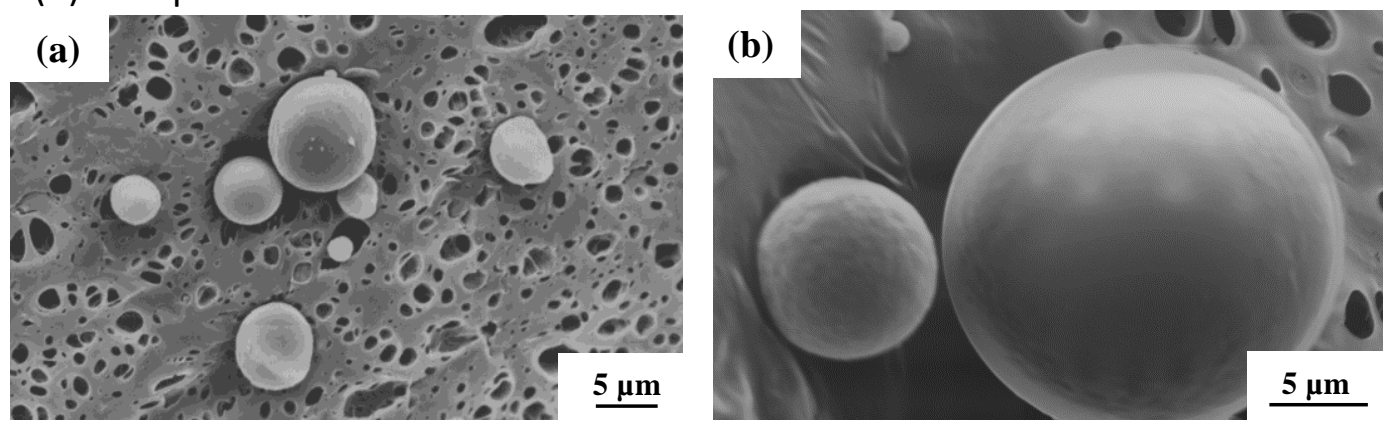

Com objetivo de obter nanopartículas de acetato de celulose, a dispersão contento as misturas de polímero e diferentes porcentagens em massa do surfactante PVA foram submetidas à agitação ultrassônica. De maneira geral, para todos os sistemas, verificou-se, inicialmente, a formação de placas. Porém, após as análises de microscópica, verificou-se a presença de nanoesferas aglomeradas. No Exp.1, onde foi utilizado a maior porcentagem de surfactante, obteve-se esferas maiores, com

diâmetro de aproximadamente 0,75 $\mu \mathrm{m}$, assim como a presença de nanoesferas de $100 \mathrm{~nm}$. Nos experimentos Exp. 2 e Exp. 3, verificou-se a presença de nanoesferas aglomeradas, com diâmetro variando de 100 a $200 \mathrm{~nm}$, sendo o sistema que utilizou 500 mg de surfactante, o que resultou em menores partículas.

Com intuito de estudar a potencialidade das micro e/ou nanopartículas de acetato de celulose como sistema para liberação controlada de 
fármaco, foi realizado um estudo preliminar de liberação com as micropartículas contendo o fármaco paracetamol. Foram obtidos espectros de absorbância em função do comprimento de onda para as microesferas de acetato de celulose com paracetamol, obtidas pelo método 1, utilizando o surfactante Tween 80, em função do tempo, em fluído de simulação gástrica $(\mathrm{pH}=1,2)$ e fluído de simulação intestinal $(\mathrm{pH}$ $=7,4)$. A partir deste gráfico foi possível construir a curva de liberação do paracetamol, Fig. 4, obtido em comprimento de onda de $240 \mathrm{~nm}$.

Figura 3. Micrografias de MEV das amostras obtidas pelo Método 2, preparadas nos experimentos: Exp.1 (a) e (b); Exp.2 (c) e (d) e Exp.3 (e) e (f).
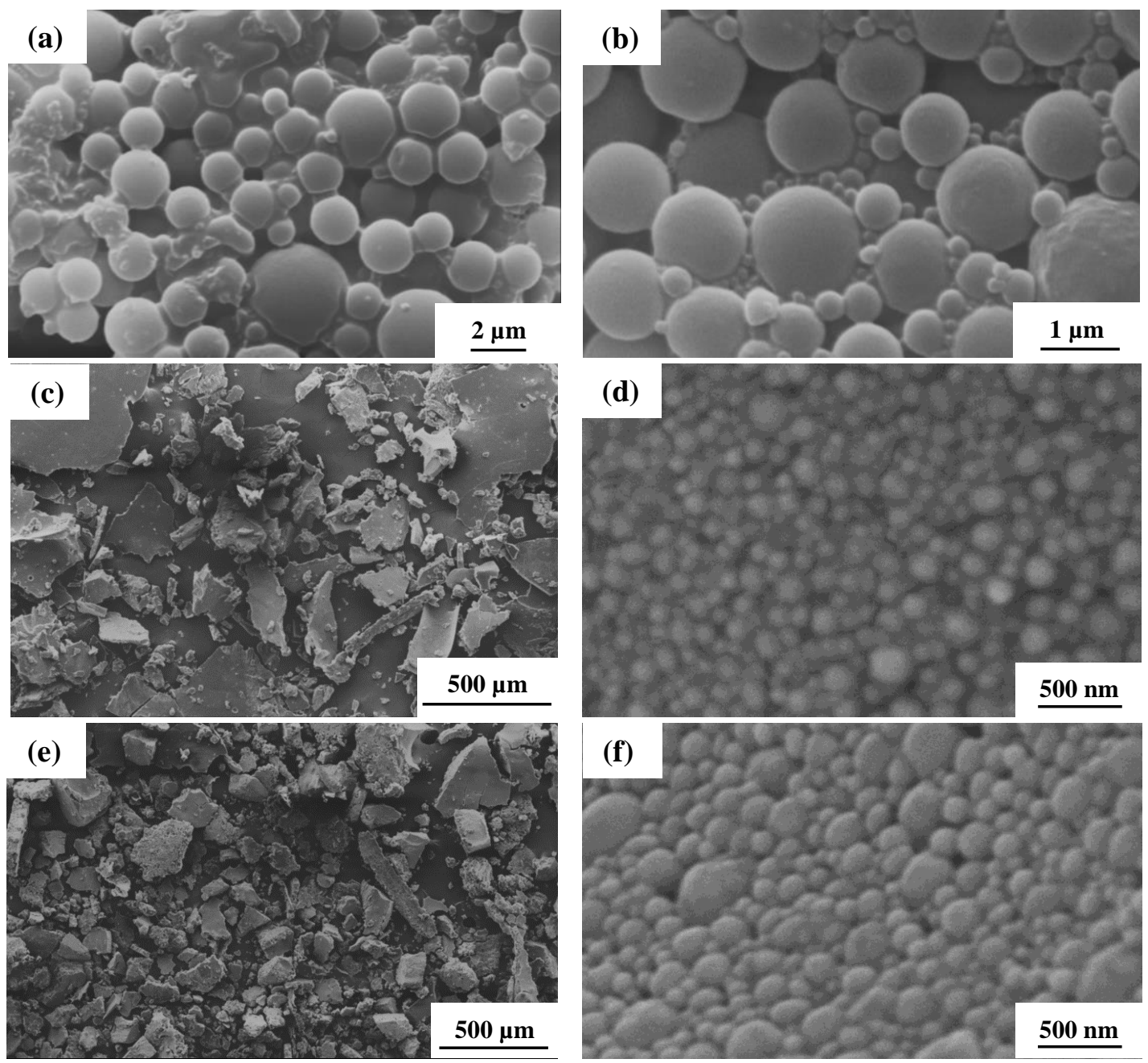
Figura 4. Curvas de liberação do paracetamol para as microesferas de $A C$, obtidas pelo método 1 : (a) fluído de simulação gástrico $(\mathrm{pH}=1,2)$ e $(b)$ fluído de simulação intestinal $(\mathrm{pH}=7,4)$.

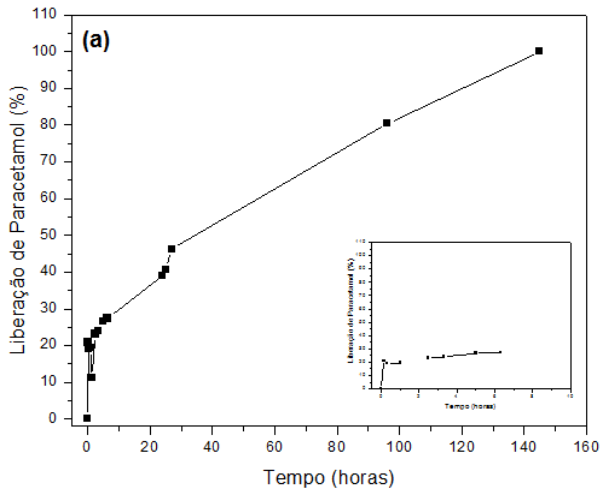

Os resultados mostram que após 15 minutos em contato com o fluído de simulação gástrica $(\mathrm{pH}=1,2)$, há liberação de 20\% de paracetamol. Em 24 horas observa-se liberação de aproximadamente 40\%, e liberação máxima em 140 horas para este sistema. Entretanto, para as microesferas em contato com o fluído de simulação intestinal $(\mathrm{pH}=7,4)$, após 15 minutos observou-se liberação de $45 \%$ de paracetamol, para 24 horas liberação de aproximadamente $90 \%$ e a liberação máxima do fármaco foi observada após 80 horas.

\section{DISCUSSÃO}

Os resultados sugerem que a presença do fármaco paracetamol favorece a formação de microesferas, com diâmetros variando de 20-250 $\mu \mathrm{m}$. Utilizando agitação ultrassônica obteve-se nanopartículas aglomeradas, com diâmetro 100 a 200 nm. O estudo da cinética de liberação do paracetamol indica que o sistema avaliado poderá apresentar um maior tempo de ação

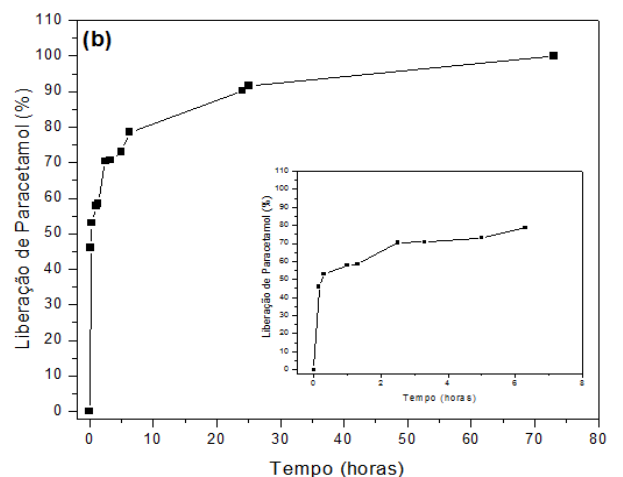

e eficiência em uma única dose, demonstrando potencialidade de aplicação das microesferas de AC como sistema para liberação controlada de anti-inflamatórios não-esteróides.

\section{REFERÊNCIAS}

ALLEN, L. V.; POPOVICH, N. G.; HOWARD, A. C. Formas Farmacêuticas e Sistemas de Liberação de Fármacos, ed. 9, p. 262-264, 2013.

BRASSEUR, N.; BRAULT, D.; COUVREUR, P. Int. J. Pharm., 70, p. 129, 1991. https://doi.org/10.1016/0378-5173(91)90172-K

CALVO, P.; VILA-JATO, J. L.; ALONSO, M. J. J. Pharm. Sci., v. 85, p. 530, 1996. https://doi.org/10.1021/js950474+

COUVREUR, P.; DUBERNET, C.; PUISIEUX, F. Eur. J. Pharm. Biopharm., v. 41, p. 2-13, 1995.

FLOWER, R. J.; VANE, J. R. Inhibition of Prostaglandin Synthetase in Brain explains the Anti-pyretic Activity of Paracetamol (4Acetamidophenol). Nature, v. 240, n. 5381, p. 410-411, 1972.

FRESTA, $M$. et al. Pefloxacine mesilate- and ofloxacin-loaded polyethylcyanoacrylate nanoparticles: characterization of the colloidal drug carrier formulation. Pharm. Sci., v. 84, p. 895, 1995. https://doi.org/10.1002/ips.2600840721 
GUTERRES, S. S. Poly(DL-lactide) nanocapsules containing diclofenac: I. Formulation and stability study. Pharma Sci., v. 11, p. 229, 2001.

HIILLERY, A. M.; TOTH, I.; FLORENCE, A. T. J. Copolymerized peptide particles (CPP). Controlled Release, v. 42, p. 65, 1996. https://doi.org/10.1016/0168-3659(96)01368-5

JUNG, T. et al. Biodegradable nanoparticles for oral delivery of peptides: is there a role for polymers to affect mucosal uptake?

Eur. J. Pharm. Biopharm., v. 50, p. 147, 2000. https://doi.org/10.1016/S0939-6411(00)00084-9

LEGRAND, P. et al. Influence of polymer behaviour in organic solution on the production of polylactide nanoparticles by nanoprecipitation. Journal of Pharmaceutics, v. 344, p. 33-43, 2007.

LOSA, C. et al. Design of new formulations for topical ocular administration: Polymeric nanocapsules containing metipranolol. Pharm. $\begin{array}{lllll}\text { Res., } & \text { v. } 10, \quad \text { p. 80, }\end{array}$ https://doi.org/10.1023/A:1018977130559

PAL, R. Rheology of simple and multiple emulsions. Current Opinion in Colloid \& Interface Science, v. 16, p. 41-60, 2011. https://doi.org/10.1016/i.cocis.2010.10.001

PARVEEN, S. et al. Nanoparticles: a boon to drug delivery, therapeutics, diagnostics and imaging. Nanomedicine: Nanotechnology, biology, and Medicine 8, p. 147-166, 2012. https://doi.org/10.1016/j.nano.2011.05.016

PINTO-ALPHANDARY, H.; ANDREMONT, A. COUVREUR, P. Targeted delivery of antibiotics using liposomes and nanoparticles: research and applications. Int. J. Antimicrob. Agents, v. 13, p. 155, 2000. https://doi.org/10.1016/S09248579(99)00121-1

RIBEIRO, S. D. et al. Cellulose triacetate films obtained from sugarcane bagasse: Evaluation as coating and mucoadhesive material for drug delivery systems. Carbohydrate Polymers, p. 764774, 2016. https://doi.org/10.1016/i.carbpol.2016.07.069

SCHAFFAZICK, S.R. et al. Caracterização e estabilidade físico-química de sistemas poliméricos nanoparticulados para administração de fármacos. Química Nova, v. 26, n. 5, p. 726737, 2003. https://doi.org/10.1590/S0100$\underline{40422003000500017}$

VILLA, A. et al. J. Design of biodegradable particles for protein delivery. Controlled Release, v. $78, \quad$ p. $15,2002$. https://doi.org/10.1016/S0168-3659(01)00486-2

YOO, H. S. et al. In vitro and in vivo anti-tumor activities of nanoparticles based on doxorubicinPLGA conjugates. Controlled Release, 68, p. 419, 2000. https://doi.org/10.1016/S01683659(00)00280-7 